\title{
Pain Perception in Taekwondo: Relationship to Injury, Experience, and Time Loss
}

(요 $\odot \odot$

Authors

Renee T. Zhao ${ }^{*}$ (D, Abdullah Kandil2*, Danh V. Nguyen ${ }^{3,4}$, Luis Campos ${ }^{5}$, Nirav H. Amin ${ }^{6}$, Eric Y. Chang ${ }^{7}$

Affiliations

1 Institute for Medical Engineering and Science, Massachusetts Institute of Technology, Cambridge, United States

2 Department of Physical Medicine and Rehabilitation, University of California Irvine, Irvine, United States

3 Institute for Clinical and Translational Science, University of California Irvine, Irvine, United States

4 Department of Medicine, University of California Irvine, Irvine, United States

5 Department of Statistics, Harvard University, Cambridge, United States

6 Orthopedic Surgery, Restore Orthopedics \& Spine Center, Orange, United States

7 Interventional Pain, Sports, Rehabilitation \& Regenerative Medicine, Restore Orthopedics \& Spine Center, Orange, United States

\section{Key words}

Sports Injury Rates, Acute Pain, Martial Arts, Return to Play

$\begin{array}{ll}\text { received } & 10.02 .2020 \\ \text { revised } & 22.04 .2020 \\ \text { accepted } & 02.05 .2020\end{array}$

\section{Bibliography}

DOI https://doi.org/10.1055/a-1168-9167

Sports Medicine International Open 2020; 4: E53-E58

(c) Georg Thieme Verlag KG Stuttgart · New York

ISSN 2367-1890

\author{
Correspondence \\ Eric Yao Chang MD \\ Restore Orthopedics \& Spine Center \\ 1120 W. La Veta Ave, Suite 300 \\ 92868 Orange, CA \\ United States \\ Tel.: + 1714598 1745, Fax: + 17149419539 \\ ericychangmd@gmail.com
}

Supporting Information for this article is available online at https://doi.org/10.1055/a-1168-9167

\section{ABSTRACT}

While the majority of sports medicine literature discusses the incidence and rehabilitation of sports injuries, there is a paucity regarding an athlete's perception of pain during these injuries. This study describes the relationship between the perception of pain from injuries in a Taekwondo collegiate conference and injury characteristics such as injury type, location, mechanism, time loss, and the athlete's competitive experience. In our study, we obtained reports from 62 Taekwondo athletes who were injured during the 2008-2009 Pacific West Taekwondo Conference collegiate season. Pain was recorded using the Numeric Rating Scale for Pain during athletes' acute injury and at two weeks, six weeks, and subsequent monthly follow-ups. Pain scores were highest for sprain/strains (mean 5.4, standard error 0.47 ) and injuries to the lower body (mean 5.6, standard error 0.36 ). By mechanism, falls (mean 5.8 , standard error 0.67 ) reported the highest levels of pain. There was a significant positive association between pain and time loss, where an increase in pain score of 1 point was associated with about 0.85 days (standard error 0.37 ) of time lost from training $(p=0.0284)$. Notably, head injuries, although potentially more devastating and attracting widespread concern, were considered less painful.

\section{Introduction}

Injury and pain are natural sequela of contact sports. An estimated 8.6 million people are injured per year through sports and recreational activities in the United States [1]. Pain from sports injuries

co-first authors. has been discussed in a few studies such as that of soccer, basketball, and rugby, but the vast majority of these studies have discussed pain as it relates to a surgical procedure or rehabilitation [2-6]. Risk factors for pain have been analyzed in several descriptive studies and cohort studies [7-9], but these studies do not discuss an athlete's perception of pain following different types of acute injury and how it relates to time loss. With increasing con- 
cern over re-injury and concussion, there is concern with returning to sport too early before underlying physiological structures have healed.

Taekwondo is a Korean martial art with over 80 million practitioners worldwide [10]. Sparring, a subspecialty of Taekwondo, is a full-contact sport that permits kicks to the head and torso and punches to the torso. World Taekwondo (WT), the regulating organization, requires that competitors wear protective gear to cover the trunk, groin, forearms, shins, gloves, head, and mouth [11]. Competitors are matched by age, rank, and weight and fight three 2-minute bouts per match. There have been numerous studies describing the frequency, severity, type, and location of Taekwondo injuries [11-13]. In a meta-analysis, Taekwondo had a competition injury rate of 79.3/1000 athlete-exposures (AE), which is higher than the rate in men's American football (35.9/1000 AE), soccer (18.8/1000 AE), and hockey (16.3/1000 AE) [11, 14]. Moreover, the prevalence of concussion in Taekwondo has been noted to be fourfold higher than American football over a 15-year span (9.4/1000 $\mathrm{AE}$ vs. 2.3/1000 AE). For all these injuries, there is no data yet on whether pain or injuries have fully resolved prior to returning to sport. In addition, pain is the body's failsafe to stop participating in a sport. Certain injuries may have higher time loss than others, yet there is minimal follow-up on these injuries and knowledge as to how pain and injury type relate to time loss.

To fill this gap, we documented pain and injury characteristics of 62 athletes in a collegiate Taekwondo season and followed up on their pain for six weeks. The purpose of this study is to investigate how the participant's self-reported pain scores change over time following an acute injury. Secondarily, this study explores the relationship of pain perception, as measured by the Numerical Rating Scale (NRS), to injury type, injury location, time loss, and athlete experience level. Finally, this study aims to identify risk factors associated with higher pain perception and inform guidelines related to injury rehabilitation and return-to-play standards.

\section{Methods}

\section{Data collection}

This is a prospective observational study that investigates Taekwondo athletes' pain following injury during the 2008-2009 Pacific West Taekwondo Conference season. Eighty athletes attended at least one of 13 competitions ( 12 scrimmage-level and 1 multistate-level) from October 2008 until March 2009. A total of 53 matches between men and 18 matches between women occurred.

The project staff included study volunteers who were present for all tournaments of the season. Whenever an injury occurred, the subject was examined by the tournament healthcare professional and the healthcare professional's exam, diagnosis, and treatment were documented by the project staff onto a paper injury form. Project staff also recorded whether the injured athlete won or lost the bout, the round in which the injury occurred, and the mechanism of injury (i. e., punch or kick and which type). Multiple injuries were filled out on multiple forms. Each athlete was assigned a unique identifier and subsequent injuries were matched to

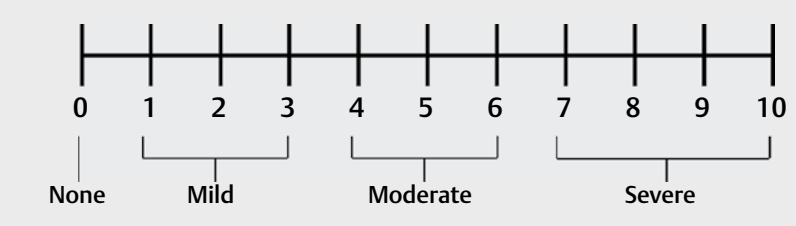

- Fig. 1 Numerical rating scale (NRS) for pain.

the same athlete. An injury was defined as a stoppage of action for medical attention and classified according to the Orchard Sports Injury Classification System (OSICS-10). This definition included both sudden-onset and acute exacerbation of slow-onset injuries, but degree of onset or history of previous injury was not recorded.

Pain was recorded using the Numeric Rating Scale for Pain (NRS) ( Fig. 1) $[15,16]$. The descriptors ranged from $0-10$, with 0 being no pain and 10 being the worst pain possible and requiring bed rest. Range 1-3 was defined as mild, 4-6 as moderate, and 7-10 as severe and prohibitive for competition. The project staff followed up with all injured athletes at two weeks and six weeks. At each interval, pain was reassessed using the NRS, which has been shown to be an efficient and accurate measure of pain assessment and monitoring $[15,17]$.

This paper is a follow-up to the senior author's previous studies of injury rates and training habits of collegiate Taekwondo athletes $[13,18]$. Approval to conduct this study was obtained from the Institutional Review Board (IRB). Informed consent was obtained from the subjects and this study followed the latest guidelines in Ethical Standards in Sport and Exercise Science Research [19]. All subjects were given a verbal description of the study and participants were assured of confidentiality and that their participation in the study would not jeopardize participation in the competition. Participation was voluntary and a written description of the study was available upon request.

\section{Statistical analysis}

Descriptive statistics were used to describe pain scores and missing data values were excluded from analyses. Linear mixed-effects (LME) models were used to compare the athletes' reported NRS pain score at the time of injury with respect to injury type, location, and types of techniques performed leading to the injury. This model accounted for correlation within subjects because some individuals had several injuries (repeated measures). Similarly, LME models were used to examine associations of age, number of months of training, and belt level (black belt level 1, 2, 3, and novice) with pain score at the time of injury. To examine pain and time-loss from training, we fitted an LME model with the main exposure as the pain score and adjusted for injury type, location, and whether individuals had multiple injuries. Missing values were excluded on a complete-case basis. Analyses were performed using SAS version 9.3 with the significance level set to 0.05 . 
- Table 1 Study participant characteristics for $\mathrm{N}=62$ athletes

\begin{tabular}{|l|l|}
\hline Variable & Mean (SD) or Count (\%) \\
\hline Age (years) & $20.90(2.47)$ \\
\hline Sex (female) & $20(32.36 \%)$ \\
\hline Weight $(\mathrm{kg})$ & $66.33(8.89)$ \\
\hline Height $(\mathrm{cm})$ & $171.85(7.79)$ \\
\hline Experience (months) & $92.24(56.27)$ \\
\hline SD: standard deviation. & \\
\hline
\end{tabular}
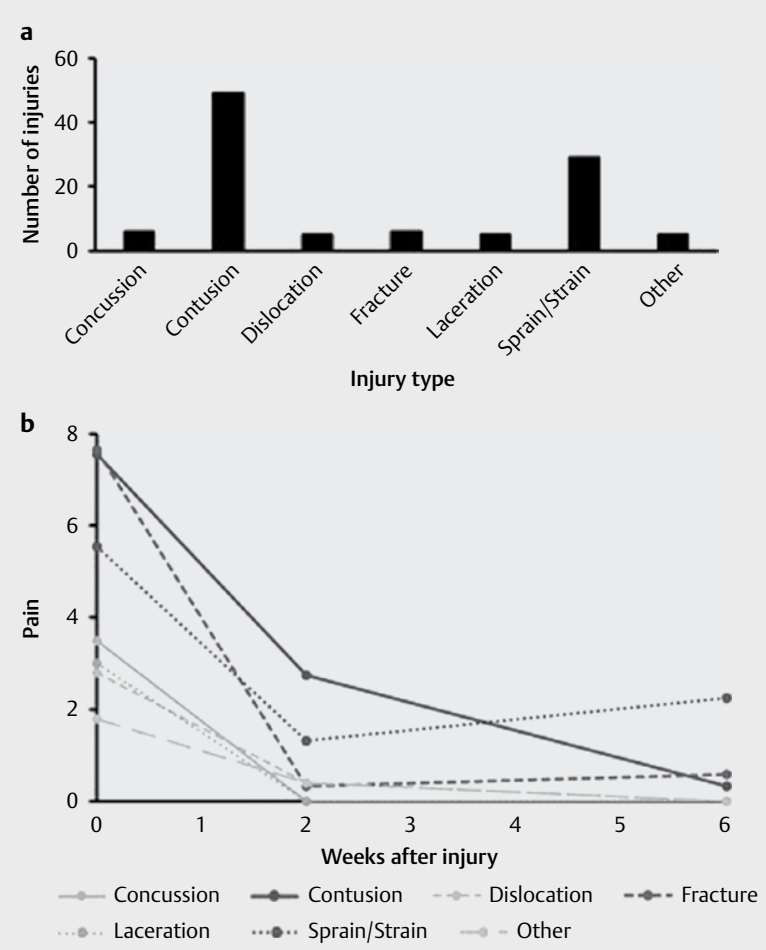

- Fig. 2 a Distribution of injuries. b Pain levels.

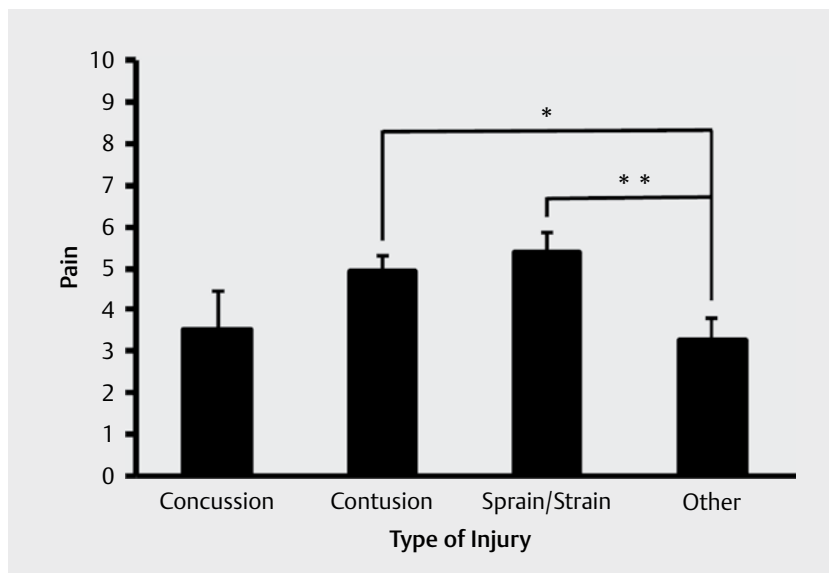

- Fig. 3 Pain according to type of injury.

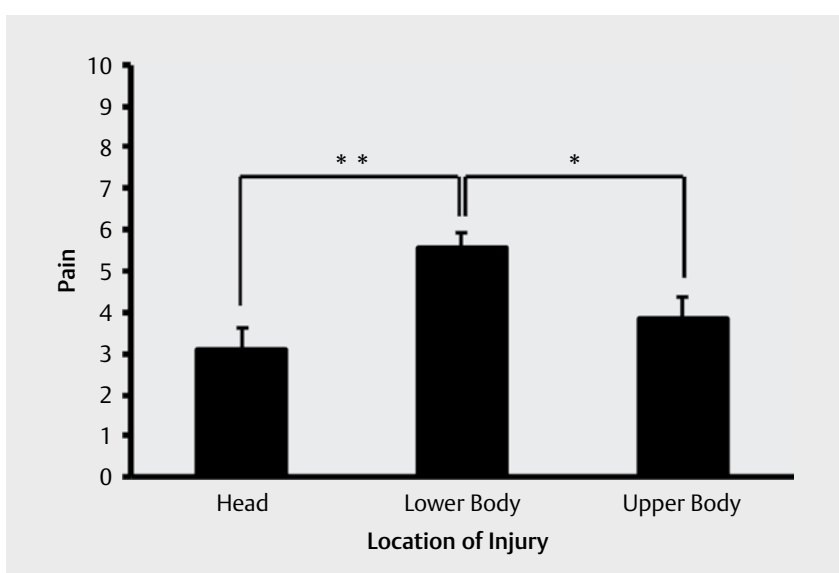

- Fig. 4 Pain according to injury location.

\section{Results}

\section{Participant characteristics}

There were 42 men and 20 women injured during our study. Mean age, weight, height, and months of experience of the athletes are depicted in - Table 1. Most participants were black belts (75.8\%). ( $\triangleright$ Fig. 1S) depicts a flow diagram of our recruitment process.

\section{Injuries and pain levels}

A total of 111 injuries were documented. The distribution of the number of injuries were: $56.45 \%$ of athletes sustained only one injury, $25.81 \%$ sustained two injuries, $8.06 \%$ sustained three injuries, $6.45 \%$ sustained four injuries, and the remaining two athletes had 6 and 7 injuries, respectively. We examined the association of reported pain levels to injury type, location, and type of techniques performed leading to the injury. - Figure 2 presents reported pain levels at the time of injury (baseline) and follow-up visits.

Our primary analysis examined the outcome pain at the time of injury. Mean level of pain scores were higher for sprain/strain (mean 5.4, standard error [SE] 0.47) and contusion (4.9 SE 0.94), which were both significantly higher than the "other" (3.2 SE 0.53) type of injury ( $p=0.0062$ and $p=0.0117$, respectively). Other group differences were not statistically significant ( $\triangleright$ Fig. 3). For location of injury (head, upper or lower body), the lower body (5.6 SE 0.36) reported the highest level of pain, followed by the upper body (3.9 SE 0.52) and head (3.1 SE 0.50); the average pain score for the lower body was significantly higher compared to upper body $(p=0.0186)$ and head $(p=0.0014)$ injuries ( $\vee$ Fig. 4 ). With respect to techniques performed leading to the injury, the mean pain score for fall (5.8 SE 0.67) and roundhouse/double kick (4.9 SE 0.33) were significantly higher than a punch (2.6 SE $0.76 ; p=0.0113$ and $p=0.0062$, respectively). No other significant differences with respect to techniques were found ( $>$ Fig. $\mathbf{5}$ ).

\section{Pain levels and experience}

We also examined athletes' reported pain score at the time of injury in association with the level of experience of the injured athlete. We found that pain was not associated with several indicators 


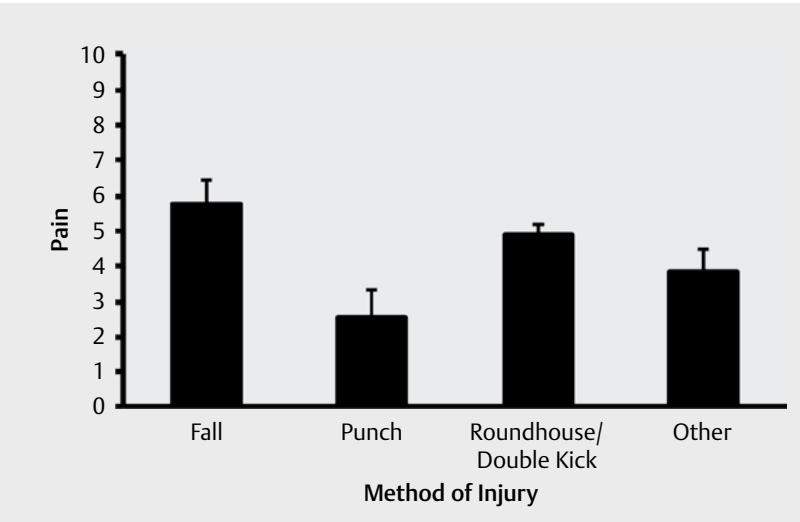

Fig. 5 Pain according to method of injury.

- Table 2 Pain and time loss from training

\begin{tabular}{|l|l|c|c|c|}
\hline Variable & Group & $\begin{array}{l}\text { Esti- } \\
\text { mate }\end{array}$ & SE & $\begin{array}{l}\text { P- } \\
\text { value }\end{array}$ \\
\hline Pain at injury & - & 0.849 & 0.372 & 0.0284 \\
\hline \multirow{2}{*}{ Location } & Head & -1.807 & 2.820 & 0.5361 \\
\cline { 2 - 5 } & Lower body & 2.819 & 2.269 & 0.2425 \\
\cline { 2 - 5 } & $\begin{array}{l}\text { Upper body } \\
\text { (reference) }\end{array}$ & - & - & - \\
\hline \multirow{7}{*}{ Type } & Concussion & 6.884 & 4.657 & 0.1576 \\
\cline { 2 - 5 } & Contusion & -3.042 & 3.005 & 0.4902 \\
\cline { 2 - 5 } & $\begin{array}{l}\text { Sprain / Strain } \\
\text { (reference) }\end{array}$ & - & - & - \\
\cline { 2 - 5 } & No & -0.177 & 1.856 & 0.9250 \\
\cline { 2 - 5 } & Yes (reference) & - & - & - \\
\hline \multirow{2}{*}{ Multiple injury } & & & \\
\hline
\end{tabular}

SE: standard error.

of experience level, including age, number of months of training, and belt level (black level 1, 2, 3, and novice).

\section{Pain and time loss from training}

Median time loss was 2 days and nearly all athletes had a time loss of less than 50 days; however, there were 5 individuals with extreme time loss (one with 90 days, three with 365 days, and one with 120 days). Thus, we examined the time that the reported pain score at injury was associated with time loss from training along with other factors of injury (location, type, and whether there were multiple injuries). There was a significant positive association between pain and time loss, where an increase in pain score of 1 point was associated with about 0.85 days (SE 0.37 ) of time lost from training $(p=0.0284)$ after controlling for injury type, location, and whether individuals had multiple injuries ( $\bullet$ Table 2 ).

\section{Discussion}

Our study is the first to investigate and document, prospectively, the amount of pain athletes reported after an injury in competition. Historically, most sports pain literature describes epidemiolo- gical data, pain tolerance, or pain prevalence, and there are few quantitative studies on the perception of pain as it relates to different athlete characteristics. Our study was able to uniquely analyze pain perception as a function of experience level, injury type, and time loss using the NRS.

We found that injuries to the lower extremities and contusions were the most common injuries by location and type, respectively, which agrees with previous findings. A recent review of Taekwondo injuries shows that contusions were the most common injury and that the lower extremity was the most vulnerable [20]. This has been shown to be true at the Olympic Games and other major tournaments $[11,13,21,22]$. Because kicks are a very common method of contact, it seems reasonable that contusions to the lower extremities are the most common injuries in Taekwondo.

We used the Numerical Rating Scale to assess the level of pain associated with Taekwondo injuries. Pain is highly subjective and therefore difficult to standardize even when using the NRS. However, we followed up with our subjects after their injuries and were able to note their declining pain levels and kept a consistent record of their subjective level of pain. We found that our most common injuries, contusions and lower extremity injuries, were also the most painful. Because we ambulate with our lower extremities and thus place large amounts of pressure on our feet, it makes sense that a lower extremity injury is more painful. Most studies have used the NRS to compare post-surgical outcomes or rehabilitation programs, and thus we were unable to find comparative usage of the NRS for acute injuries.

Interestingly, potentially devastating injuries such as concussion did not rate as painful to the athletes in our study. However, these head injuries may be more concerning because of concerns about long-term damage, and more recently, chronic traumatic encephalopathy (CTE) $[23,24]$. Concussion has been shown to be four times more prevalent in Taekwondo than in football [21], although many concussed Taekwondo athletes return to play within a few days [25], which we found to occur among our athletes as well. Moreover, we were able to note that our athletes' concussive pain fully dissipated within two weeks, adhering to current return-to-play protocols that rely on the athlete feeling asymptomatic with activity [26]. However, recent research shows that complete recovery of underlying anatomical structures lags behind clinical symptom resolution even after one year [27]. As our study shows, concussive pain may be a poor indicator of injury resolution because most people do not report pain at two weeks post-injury.

There is a need to clarify what concussive pain is, because it often does not feel acutely painful the same way a bruise does. Neck pain may be the most commonly conceived notion of pain, but not all concussed patients develop neck pain. In recent studies, only $68.4 \%$ of patients in the emergency room presented with neck pain within three days of injury, and confusingly, up to $20 \%$ of varsity collision-sport athletes were found to have pre-season neck pain $[28,29]$. Furthermore, a myriad of concussion symptoms are closely intertwined with and mistaken for other conditions such as depression, anxiety, and PTSD. Because previous concussions are a risk factor for future concussions, it may be that many athletes return to play without fully recovering from a previous concussion [26]. This may highlight a lack of clarity in mapping concussive pain 
to symptom resolution, which may lead athletes to return to play too soon.

Our study suggests that although most Taekwondo competition injuries appear mild, many athletes elect to return to sport before their pain has fully resolved. At two weeks, $39.6 \%$ of our athletes still had pain, yet the median time loss was only two days. Our time-loss rates are comparable to previous studies which show that up to $74 \%$ of competition injuries result in $<1$ day of time loss, and most of the remaining injuries result in a time loss of 2-7 days [30]. Sprains/strains are common in Taekwondo [11], yet in our study, pain actually increased after six weeks. Furthermore, our linear mixedeffects model showed that injury location, type, and presence of multiple injuries did not affect time loss, suggesting that players may return soon after injury regardless of the type of injury they have experienced. Traditionally, it takes contusions four to seven days to heal and fractures four to eight weeks to heal, yet time loss was unaffected by injury type, showing how pain may ambiguously map to underlying physical damage.

It is crucial to standardize return-to-play procedures for athletes, keeping in mind the limitations of using pain as a gatekeeper for play. Acute pain is the body's physiological monitor for whether to restart an activity once the pain is tolerable or subsides completely, but if it is tolerable, we must ensure that there is no damage to the underlying structures. Further research needs to be done to determine the amount of time for optimal injury healing that is easily measured via radiographical evidence, the development of biomarkers that measure pain, or recovery from injury, or psychometric analysis.

\section{Strengths and limitations}

Our study was able to classify the types, locations, and methods of injury and compare them to the severity of pain according to the NRS during the 2008-2009 Pacific West Taekwondo Conference season. We followed up with our subjects until their injuries resolved. Limitations to our study include small sample size and lack of investigation as to mechanistically why lower extremity injuries were most painful. Perhaps a future study can use a larger sample size of athletes across the martial arts or sports spectrum or include a meta-analysis from multiple competitions.

\section{Conclusion}

Our study was able to classify the types, locations, and method of injury and compare them to the severity of pain according to the NRS. The data and multiple follow-ups showed that lower extremity contusions were the most painful and common injury. Importantly, head injuries, although potentially more devastating and of widespread public concern, were less painful.

\section{Funding}

This work was supported in part by grant K12 HD001097 (E.C.) and grant UL1 TR000153 (E.C., D.V.N.) from the National Center for Advancing Translational Sciences through the Biostatistics, Epidemiology and Research Design Unit of UC Irvine Institute for Clinical Translational Science.

\section{Conflict of interest}

The authors declare that they have no conflict of interest.

\section{References}

[1] Sheu Y, Chen LH, Hedegaard H. Sports-and recreation-related injury episodes in the United States, 2011-2014. Natl Health Stat Report 2016; 99: 1-12

[2] Oztekin HH, Boya H, Ozcan O et al. Pain and affective distress before and after ACL surgery: A comparison of amateur and professional male soccer players in the early postoperative period. Knee 2008; 15 : 368-372

[3] Ghasemi M, Bagheri H, Olyaei G et al. Effects of cyclic static stretch on fatigue recovery of triceps surae in female basketball players. Biol Sport 2013; 30: 97-102

[4] Serinken MA, Gencoglu C, Kayatekin BM. The effect of eccentric exercise-induced delayed-onset muscle soreness on positioning sense and shooting percentage in wheelchair basketball players. Balkan Med J 2013; 30: 382-386

[5] Mohseni-Bandpei MA, Keshavarz R, Minoonejhad H et al. Shoulder pain in Iranian elite athletes: The prevalence and risk factors. J Manipulative Physiol Ther 2012; 35: 541-548

[6] Iwamoto ], Abe H, Tsukimura Y et al. Relationship between radiographic abnormalities of lumbar spine and incidence of low back pain in high school rugby players: A prospective study. Scand J Med Sci Sports 2005; 15: 163-168

[7] Nixon HL. The relationship of friendship networks, sports experiences, and gender to expressed pain thresholds. Sociol Sport J 2016; 13: $78-86$

[8] Miller C, Newton SE. Pain perception and expression: The influence of gender, personal self-efficacy, and lifespan socialization. Pain Manag Nurs 2006; 7: 148-152

[9] Yaffe M. Sports injuries. Psychological aspects. Br] Hosp Med 1983; 2 : 224-232

[10] World Taekwondo. Available from: worldtaekwondo.org

[11] Lystad RP, Pollard H, Graham PL. Epidemiology of injuries in competition Taekwondo: A meta-analysis of observational studies. J Sci Med Sport 2009; 12: 614-621

[12] Beis K, Pieter W, Abatzides G. Taekwondo techniques and competition characteristics involved in time-loss injuries. J Sport Sci Med 2007; 6: 45-51

[13] Natalia Covarrubias SB, Campos L, Nguyen D et al. The relationship between Taekwondo training habits and injury: A survey of a collegiate Taekwondo population. Open Access J Sport Med 2015; 6: 1-7

[14] Hootman JM, Dick R, Agel J. Epidemiology of collegiate injuries for 15 sports: Summary and recommendations for injury prevention initiatives. J Athl Train 2007; 42: 311-319

[15] Hawker GA, Mian S, Kendzerska T et al. Measures of adult pain: Visual Analog Scale for Pain (VAS Pain), Numeric Rating Scale for Pain (NRS Pain), McGill Pain Questionnaire (MPQ), Short-Form McGill Pain Questionnaire (SF-MPQ), Chronic Pain Grade Scale (CPGS), Short Form-36 Bodily Pain Scale (SF-36 BPS), and Measure of Intermittent and Constant Ostioarthritis Pain (ICOAP). Arthritis Care Res (Hoboken) 2011; 63: S240-S252

[16] Farrar JT, Young JP, LaMoreaux L et al. Clinical importance of changes in chronic pain intensity measured on an 11-point numerical pain rating scale. Pain 2001; 96: 410-411

[17] Chaffee A, Yakuboff M, Tanabe T. Responsiveness of the VAS and McGill pain questionnaire in measuring changes in musculoskeletal pain. J Sport Rehabil 2011; 20: 250-255 
[18] Chang E, Fish DE, Hsieh S et al. Poster 130: Epidemiology and mechanism of injury in a major collegiate Taekwondo Tournament. PM\&R 2009; 1: S160-S161

[19] Harriss D, Macsween A, Atkinson G. Ethical standards in sport and exercise science research: 2020 update. Int J Sports Med 2019; 40: 813-817

[20] Thomas RE, Thomas BC, Vaska MM. Injuries in Taekwando: Systematic review. Phys Sportsmed 2017; 45: 372-390

[21] Pieter W, Fife GP, O'sullivan DM. Competition injuries in Taekwondo: A literature review and suggestions for prevention and surveillance. $\mathrm{Br}$ ] Sports Med 2012; 46: 485-491

[22] Kazemi M, Pieter W. Injuries at a Canadian National Taekwondo Championships: A prospective study. BMC Musculoskelet Disord 2004; 5: 22

[23] Ling $\mathrm{H}$, Hardy J, Zetterberg $\mathrm{H}$. Neurological consequences of traumatic brain injuries in sports. Mol Cell Neurosci 2015; 66: 114-122

[24] Makdissi M, Patricios J. Comprehending concussion: Evolving and expanding our clinical insight. Br J Sports Med 2015; 49: 1029-1030
[25] Schlüter-Brust K, Leistenschneider P, Dargel ] et al. Acute injuries in Taekwondo. Int J Sports Med 2011; 32: 629-634

[26] West TA, Marion DW. Current recommendations for the diagnosis and treatment of concussion in sport: A comparison of three new guidelines. J Neurotrauma 2014; 31: 159-168

[27] Churchill NW, Hutchison MG, Graham S] et al. Mapping brain recovery after concussion: From acute injury to 1 year after medical clearance. Neurology 2019; 93: 1980-1992

[28] King JA, McCrea MA, Nelson LD. Frequency of primary neck pain in mild traumatic brain injury/concussion patients. Arch Phys Med Rehabil 2020; 101: 89-94

[29] Shehata N, Wiley JP, Richea S et al. Sport concussion assessment tool: Baseline values for varsity collision sport athletes. Br J Sports Med 2009; 43: 730-740

[30] Sherrill PM. Martial-art injuries at a major Midwest tournament: Results of a cumulative two-year study and a comparison with other recent studies. J Osteopath Sport Med 1989; 3: 9-11 